\title{
Eficácia de atrazine sobre populações de Paspalum virgatum L. e seletividade em duas variedades de pastagem
}

\author{
Effectiveness of atrazine on populations of capim navalha and selectivity \\ of two varieties of pastagem
}

Vanessa Takeshita ${ }^{*}$, Kassio Ferreira Mendes ${ }^{1}$, Miriam Hiroko Inoue ${ }^{2}$, Ana Carolina Dias Guimarães ${ }^{3}$

Resumo - O Paspalum virgatum L. é uma planta daninha comum de pastagens e possui difícil controle, principalmente devido sua semelhança com as forrageiras. As técnicas convencionais como gradagem e replantio da pastagem têm sido insatisfatórias no controle desta planta daninha. O objetivo deste trabalho foi avaliar o controle do capim-navalha ( $P$. virgatum L.) à atrazine aplicada em pré-emergência, por meio de curva dose-resposta e a seletividade deste herbicida nas pastagens de Brachiaria brizantha cv. Marandu e Panicum maximum cv. Mombaça. Foram realizados três experimentos independentes, com cada espécie, em dois tipos de solo (Latossolo Vermelho Amarelo distrófico - LVAd de textura franco-argilosa e Argissolo Vermelho Amarelo distrófico - PVAd de textura franca), utilizando a escala percentual de notas para verificar o controle do capim-navalha e a fitotoxicidade nas forrageiras e massa seca no final dos experimentos. A atrazine controlou o capim-navalha, desde os 15 dias após a aplicação (DAA), nas doses abaixo da recomendação do fabricante (250 e $500 \mathrm{~g} \mathrm{ha}^{-1}$ i.a.). Contudo, apesar dos sintomas de fitotoxicidade causados pela atrazine, houve retomada de crescimento aos 60 DAA tanto para $B$. brizantha $\mathrm{cv}$. Marandu quanto para $P$. maximum cv. Mombaça. Houve menor fitotoxicidade às pastagens no solo de textura franco-argilosa em relação ao solo de textura franca. A atrazine proporcionou controle eficiente do $P$. virgatum L. (500 $\mathrm{g} \mathrm{ha}^{-1}$ i.a.) quando aplicada em pré-emergência e seletividade para $B$. brizantha cv. Marandu ( $<2.000 \mathrm{~g} \mathrm{ha}^{-1}$ i.a.) e P. maximum cv. Mombaça $\left(<500 \mathrm{~g} \mathrm{ha}^{-1}\right.$ i.a. $)$.

Palavras-chave: Paspalum virgatum L., controle químico, fitotoxicidade, resposta biológica, triazina

Abstract - Paspalum virgatum L. is a grass weed common in pasture and has difficult control, mainly due to its similarity to forage. Conventional techniques such as grazing and replanting of pasture have been unsatisfactory in the control of this weed. The objective of this work was to evaluate the control of the razor-blade grass ( $P$. virgatum L.) at the pre-emergence atrazine, by means of a dose-response curve and the selectivity of this herbicide in the pastures of Brachiaria brizantha cv. Marandu and Panicum maximum cv. Mombasa. Three independent experiments were carried out with each species on two types of soils (Dystrophic Yellow Red Latosol - LVAd with loamy clay texture and

Recebido: Janeiro 31, 2018. Aceito: Setembro 15, 2018.

${ }^{1}$ Centro de Energia Nuclear na Agricultura - CENA, Universidade de São Paulo - USP, Av. Centenário, 303, São Dimas, CEP 13416-000, Piracicaba, SP, Brasil. E-mail: vanessa_takeshita@hotmail.com; kassio_mendes_06@hotmail.com

${ }^{2}$ Universidade do Estado de Mato Grosso - UNEMAT, Campus Tangará da Serra, Tangará da Serra, MT, Brasil. E-mail: miriamhinoue@hotmail.com

${ }^{3}$ Universidade do Estado de Mato Grosso - UNEMAT, Campus Alta Floresta, Alta Floresta, MT, Brasil. E-mail: acrdias@unemat.br 
Dystrophic Yellow Red Argisol - loamy texture PVAd), using the percentage grading scale to verify the control of the and herbage phytotoxicity and dry mass at the end of the experiments. Atrazine controlled the razor blade from 15 days after application (DAA) at doses below the manufacturer's recommendation (250 and $500 \mathrm{~g} \mathrm{ha}^{-1}$ i.a.). However, despite the phytotoxicity symptoms caused by atrazine, there was a resumption of growth at 60 DAA for both $B$. brizantha cv. Marandu and P. maximum cv. Mombasa. There was less phytotoxicity to pastures in the soil of loamy clay texture than the loamy texture soil. The atrazine provided efficient control of P. virgatum L. ( $500 \mathrm{~g} \mathrm{ha}^{-1}$ i.a. $)$ when applied in pre-emergence and selectivity to $B$. brizantha $\mathrm{cv}$. Marandu $\left(<2,000 \mathrm{~g} \mathrm{ha}^{-1}\right.$ i.a. $)$ and P. maximum cv. Mombasa ( $<500 \mathrm{~g} \mathrm{ha}^{-1}$ i.a.).

Keywords: Paspalum virgatum L., chemical control, phytotoxicity, biological response, triazine

\section{Introdução}

O Brasil possui o maior rebanho bovino comercial do mundo, com mais de 218 milhões de cabeças de gado, sendo o Estado de Mato Grosso o detentor do maior rebanho $(13,9 \%$ do total brasileiro), com mais de 30 milhões de cabeças (Produção da Pecuária Municipal, 2016). Sendo a maior parte da carne produzida no Brasil de origem em animais mantidos exclusivamente a pasto. No entanto, na Amazônia legal brasileira, da qual o Estado de Mato Gross está inserido, possui cerca de $50 \%$ das áreas de pastagens estejam degradadas ou em processo de degradação (Dias-Filho, 2011). Segundo este mesmo autor, o processo de degradação pode ser inicialmente caracterizado pela mudança na composição botânica da pastagem, pelo aumento na proporção de plantas daninhas e da diminuição na proporção de forrageira desejada.

Dentre as plantas daninhas que ocorrem nas pastagens destaca-se o capim-navalha (Paspalum virgatum L.), que é uma gramínea perene, encontrada no Brasil em todos os Estados das regiões Norte e Centro-Oeste, além do Maranhão, Pernambuco, São Paulo e Paraná (Valls e Oliveira, 2012). Esta planta daninha ocupa posição de destaque dentre as plantas daninhas de pastagens de difícil controle, devido a sua semelhança morfológica, fisiológica e bioquímica com as forrageiras, rebrota da touceira ou reinfestação da área por sementes. De modo que o controle por métodos convencionais como, gradagem e semeadura de novas forrageiras têm sido frustrantes, de acordo com Andrade et al. (2012). O capim-navalha é considerado uma planta daninha de pastagem devido sua baixa palatabilidade e da alta capacidade de multiplicação (Andrade et al., 2012). Como alternativas de controle, há relatos na literatura do controle químico do capim-navalha em pré-emergência com trifluralin $\left(600 \mathrm{~g} \mathrm{ha}^{-1}\right.$ i.a. $)$ aplicado em pré-plantio incorporado, inibindo sua germinação (Sistachs et al., 1982), com atrazine em pré-emergência (3000 $\mathrm{g} \mathrm{ha}^{-1}$ i.a.) (Sistachs e León, 1987) e, na pós-emergência temos haloxyfop-methyl (300 $\mathrm{g} \mathrm{ha}^{-1}$ i.a.) (De la Cruz et al., 1990) e glyphosate (360 $\mathrm{g} \mathrm{ha}^{-1}$ e.a.) 54 dias após a semeadura, com 6 folhas completamente expandidas, obtendo $100 \%$ de controle (Silva et al., 2017).

Na região norte do Estado de Mato Grosso, a prática de reforma de pastagens, principalmente devido a ocorrência da morte súbita das pastagens, tem sido fortemente utilizada. A substituição das pastagens pela cultura do milho é uma alternativa para amortizar os custos da reforma (Kluthcouski e Yokoyama, 2003), podendo ser utilizada em sistema de Integração Lavoura-Pecuária (ILP) ou substituída novamente pelo plantio das forrageiras após seu ciclo anual de cultivo. Neste sentido, a atrazine, por ser um herbicida seletivo para a cultura do milho, é promissora na seletividade para gramíneas forrageiras (Loch e Harvey, 1997) e um produto potencial no controle do capim-navalha (Sistachs e León, 1987). 
A atrazine é utilizada em pré e pós-emergência inicial, no controle de dicotiledôneas e algumas gramíneas, pertence ao grupo das triazinas, sendo inibidor do Fotossistema II (Girotto et al., 2011). Os sintomas de fitotoxicidade apresentados com a aplicação deste herbicida são clorose seguida de necrose, devido a interrupção no fluxo de elétrons da fotossíntese, levando a morte da planta (Akbulut e Yigit, 2010). Pode inibir também a germinação de sementes de plantas daninhas (Merini et al., 2009). No solo, apresenta baixo potencial de sorção (Archangelo et al., 2010; Scorza Júnior e Rigitano, 2012; Carvalho et al., 2013) e, é moderadamente adsorvida pelos coloides de argila e da matéria orgânica (Silva et al., 2007).

De acordo com Binet et al. (2006), a atrazine possui alta afinidade molecular com complexos organo-minerais do solo, sendo a sorção correlacionada com o teor de carbono orgânico presente no solo. Essa interação herbicida-solo interferente diretamente na biodisponibilidade do herbicida. De modo que a biodisponibilidade da atrazine é reduzida quando sorvida a matéria orgânica do solo (Binet et al., 2006). Assim, a eficácia de controle das plantas daninhas e seletividade de culturas podem ser afetados pela característica dos solos, principalmente pelo teor de matéria orgânica. Neste contexto, o objetivo deste trabalho foi avaliar a suscetibilidade do capim-navalha $(P$. virgatum $\mathrm{L}$.) a atrazine em dois solos de textura distintas e avaliar os efeitos fitotóxicos deste herbicida sobre as duas pastagens (Brachiaria brizantha cv. Marandu e Panicum maximum cv. Mombaça) mais utilizadas na região norte do Mato Grosso.

\section{Material e métodos}

Os experimentos foram realizados em casa de vegetação (9॰53'49, 1'S 56 05'37,1'O) no período de fevereiro a abril de 2016. Foram utilizados dois tipos de solo, um Latossolo Vermelho Amarelo distrófico (LVAd) de textura franco-argilosa e um Argissolo Vermelho Amarelo distrófico (PVAd) de textura franca. A correção de $\mathrm{pH}$ nos solos foi realizada elevando a saturação por bases para 60\% com calcário filler (PRNT 95\%) e de fertilidade com ureia, $\mathrm{P}_{2} \mathrm{O}_{5}$ e $\mathrm{K}_{2} \mathrm{O}$, de acordo com a interpretação da análise de solo (Tabela 1) e as recomendações de Vilela et al. (2004).

Foram realizados três experimentos independentes, para cada uma das três espécies de gramíneas $(P$. virgatum L. B. brizantha cv. Marandu e $P$. maximum cv. Mombaça). As sementes de $P$. virgatum $\mathrm{L}$. foram coletadas em áreas de pastagens infestadas, sendo estas identificadas e os propágulos acondicionados em sacos de papel, em local seco, à temperatura ambiente. As sementes de capim-navalha foram submetidas à quebra de dormência em ácido sulfúrico $(100 \mathrm{~mL})$, por aproximadamente $15 \mathrm{~min}$, de acordo com a escarificação química testada

Tabela 1. Propriedades químicas e físicas dos solos utilizados nos experimentos (Alta Floresta - MT, 2016).

\begin{tabular}{|c|c|c|c|c|c|c|c|c|c|c|}
\hline \multirow{2}{*}{ Solo } & pH & MO & $\mathbf{P}^{1}$ & $\mathbf{K}$ & $\mathbf{C a}$ & Mg & $\mathbf{A l}$ & CTC & $\mathbf{V}$ & $\mathbf{M}$ \\
\hline & $\mathrm{H}_{2} \mathrm{O}$ & $\mathrm{g} \mathrm{kg}^{-3}$ & $\mathrm{mg} \mathrm{dm}^{-3}$ & \multicolumn{5}{|c|}{$\mathrm{mmol}_{\mathrm{c}} \mathrm{dm}^{-3}$} & \multicolumn{2}{|c|}{$\%$} \\
\hline LVAd franco argiloso & 4,7 & 18,7 & 1,1 & 11,7 & 0,2 & 0,10 & 0,92 & 4,6 & 7,2 & 73,6 \\
\hline PVAd franco & 5,0 & 14,2 & 0,9 & 31,2 & 0,84 & 0,45 & 0,48 & 3,8 & 38,5 & 25,9 \\
\hline \multirow{2}{*}{ Solo } & \multicolumn{3}{|c|}{ Argila } & \multicolumn{4}{|c|}{ Silte } & \multicolumn{3}{|c|}{ Areia } \\
\hline & \multicolumn{10}{|c|}{$\%$} \\
\hline LVAd franco argiloso & \multicolumn{3}{|c|}{59,0} & \multirow{2}{*}{\multicolumn{4}{|c|}{5,7}} & \multicolumn{3}{|c|}{35,3} \\
\hline PVAd franco & \multicolumn{3}{|c|}{28,8} & & & & & \multicolumn{3}{|c|}{61,2} \\
\hline
\end{tabular}

${ }^{1} \mathrm{Pe} \mathrm{K} \mathrm{K}^{+}$obtidos por meio do extrator Melich1. Fonte: LASAF - Laboratório de solos e análise foliar (Alta Floresta - MT). $\mathrm{MO}=$ Matéria orgânica; $\mathrm{CTC}=$ Capacidade de troca catiônica; $\mathrm{V}=$ Saturação por bases; $\mathrm{M}=$ Saturação por alumínio. 
por Silva et al. (2018), para atingir o mínimo de $60 \%$ de germinação. Também foram utilizadas sementes de $B$. brizantha cv. Marandu (pureza: 60\%, germinação: $80 \%$ e valor cultural: $48 \%$ ) e $P$. maximum cv. Mombaça (pureza: 50\%, germinação: $78 \%$ e valor cultural: 39 ).

$\mathrm{O}$ delineamento experimental adotado para cada experimento foi o de blocos ao acaso em esquema fatorial $8 \times 2$, sendo 8 o número de doses (8D - $16.000 \mathrm{~g} \mathrm{ha}^{-1}$ i.a., 4D - $8.000 \mathrm{~g} \mathrm{ha}^{-1}$ i.a., 2D - $4.000 \mathrm{~g} \mathrm{ha}^{-1}$ i.a., D - $2.000 \mathrm{~g} \mathrm{ha}^{-1}$ i.a., 1/2D - $1.000 \mathrm{~g} \mathrm{ha}^{-1}$ i.a., 1/4D - $500 \mathrm{~g} \mathrm{ha}^{-1}$ i.a., $1 / 8 \mathrm{D}$ - $250 \mathrm{~g} \mathrm{ha}^{-1}$ i.a. e ausência de dose, em que D é a dose recomendada de atrazine) e 2 solos, com quatro repetições. De modo que cada unidade experimental foi constituída de um vaso plástico com capacidade de $4 \mathrm{mg} \mathrm{dm}^{-3}$, contendo 64 unidades experimentais por experimento.

As sementes foram semeadas no dia 20 de fevereiro de 2016, sendo 10 sementes por vaso, tanto do capim navalha, quanto das forrageiras, cada espécie no seu respectivo experimento. A semeadura foi realizada $8 \mathrm{~h}$ antes da aplicação em pré-emergência do herbicida, com uso de pulverizador costal pressurizado com $\mathrm{CO}_{2}$, mantendo-se a pressão constante de $275.79 \mathrm{kPa}$, ponta de pulverização tipo leque, com jato plano, modelo AXI 110-02, com volume de calda de $200 \mathrm{~L} \mathrm{ha}^{-1}$. No momento da aplicação, a condição climática foi de temperatura de $35^{\circ} \mathrm{C}$, umidade de $41 \%$, velocidade do vento de $0,8 \mathrm{Km} \mathrm{h}^{-1}$ na direção sudeste.

As avaliações foram realizadas aos 15, 30 e 60 dias após a aplicação (DAA) da atrazine no experimento com $P$. virgatum $\mathrm{L}$. foram dadas notas visuais de controle, sendo $0 \%$ para o não controle e $100 \%$ para controle total (ALAM, 1974), nos ensaios de fitotoxicidade das forrageiras (B. brizantha $\mathrm{cv}$. Marandu e $P$. maximum cv. Mombaça) utilizou-se a escala do EWRC (1964) e ao final dos experimentos foram feitas massa seca da parte aérea. A massa seca foi obtida a partir da colheita do material vegetal da parte aérea das plantas nas unidades experimentais, com posterior secagem em estufa de circulação forçada de ar a $70^{\circ} \mathrm{C}$ por $96 \mathrm{~h}$, sendo corrigidos os valores de massa seca para porcentagem em relação à massa seca total obtida nos tratamentos testemunhas, sem aplicação do herbicida (Guimarães et al., 2016).

Inicialmente, para cada experimento, os dados foram submetidos à análise da variância (ANOVA) com aplicação do teste F. Quando o teste $\mathrm{F}$ foi significativo, as variáveis de controle e fitotoxicidade foram ajustadas a modelos de regressão não lineares do tipo log-logístico (metodologia de curva-dose-resposta), com o auxílio do software SigmaPlot ${ }^{\circledR}$ (versão 10.0.1, 2007, para Windows, Systat Software Inc., Point Richmond, CA, EUA).

Para a variável controle, adotou-se o modelo proposto por Streibig et al. (1993) (Equação 1):

$$
y=\frac{a}{\left[1+\left(\frac{x}{b}\right)^{c}\right]}
$$

em que: $y$ é a porcentagem de controle; $x$ é a dose do herbicida; e $a, b$ e $c$ são parâmetros da curva, de modo que $a$ é a diferença entre o ponto máximo e mínimo da curva, $b$ é a dose que proporciona $50 \%$ de resposta da variável e $c$ é a declividade da curva.

Para a variável massa seca, adotou-se o modelo proposto por Seefeldt et al. (1995) (Equação 2):

$$
y=\operatorname{Pmín}+\frac{a}{\left[1+\left(\frac{x}{b}\right)^{c}\right]}
$$

em que: $y$ é a porcentagem residual da massa seca; $x$ é a dose do herbicida; e Pmín, $a, b$ e $c$ são parâmetros da curva, de modo que Pmín é o limite inferior da curva (ponto mínimo), $a$ é a diferença entre o ponto máximo e mínimo da curva, $b$ é a dose que proporciona $50 \%$ de resposta da variável e $c$ é a declividade da curva. 


\section{Resultados e discussão}

Para as fontes de variação dose e solos, no experimento com o capim-navalha, não houve diferença significativa de controle aos $15(\mathrm{~F}=1,0$; $p>0,05)$ e $30 \mathrm{DAA}(\mathrm{F}=1,0 ; p>0,05)$, não justificando o desdobramento da interação. Aos 60 DAA houve interação entre as fontes de variação dose e solo para controle $(\mathrm{F}=43,39 ; p<0,05)$ e massa seca ( $\mathrm{F}=131,41 ; p<0,05)$, entretanto, não houve possibilidade de ajuste dos dados em curva dose-resposta, devido à alta suscetibilidade da planta daninha à atrazine, como verificado na Figura 1, para todos os dias de avaliação. Todas as
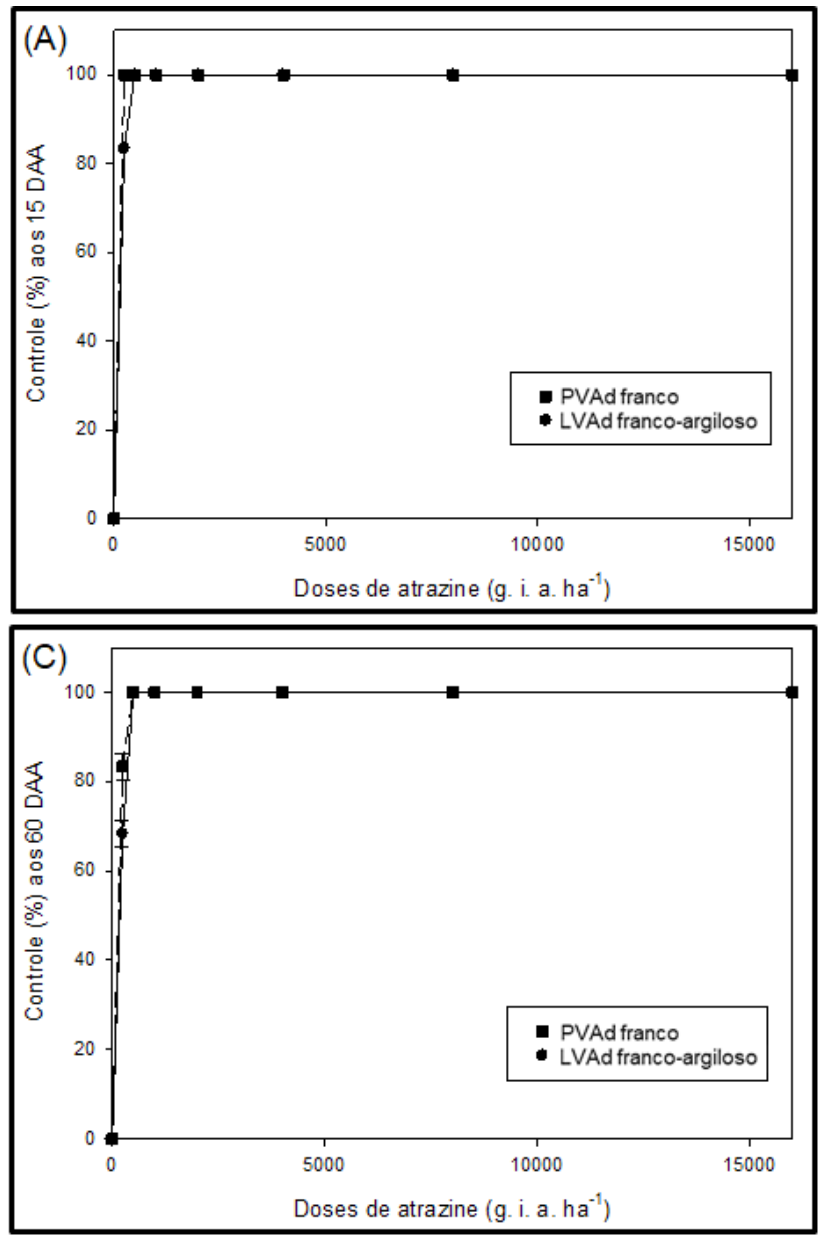

notas de controle apresentadas para o $P$. virgatum L. foram altas (acima de 80\%) desde a menor dose aplicada (250 $\mathrm{g} \mathrm{ha}^{-1}$ i.a.), aos 15 e 30 DAA de avaliação, para ambos os solos.

Semelhantes aos resultados encontrados para o controle de capim-navalha neste trabalho, com alta porcentagem de controle da planta daninha na dose recomendada da atrazine, bem como em menores doses, Sistachs e León (1987) observaram controle de $85 \%$ na dose de $2.000 \mathrm{~g} \mathrm{ha}^{-1}$ i.a. (dose recomendada de atrazine) e $100 \%$ na dose de $3.000 \mathrm{~g} \mathrm{ha}^{-1}$ i.a. para $P$. virgatum L., encontrando mais de $50 \%$ de controle do capim-navalha na dose de $1.500 \mathrm{~g} \mathrm{ha}^{-1}$ i.a. de atrazine.
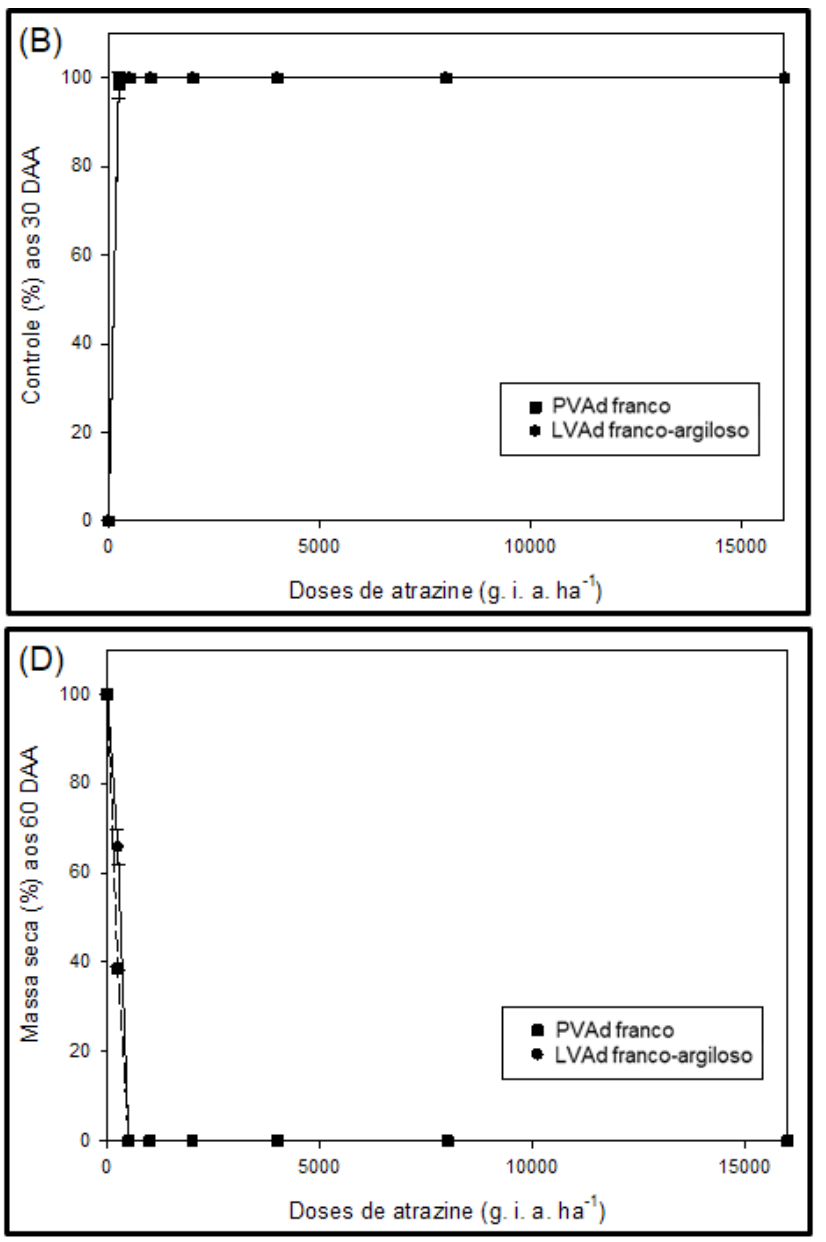

Figura 1. Controle (\%) de $P$. virgatum submetidas a diferentes doses de atrazine, em solo franco e franco argiloso, aos 15 (A), 30 (B) e 60 (C) dias após a aplicação (DAA) e massa seca aos 60 DAA (D) (Alta Floresta - MT, 2016). 

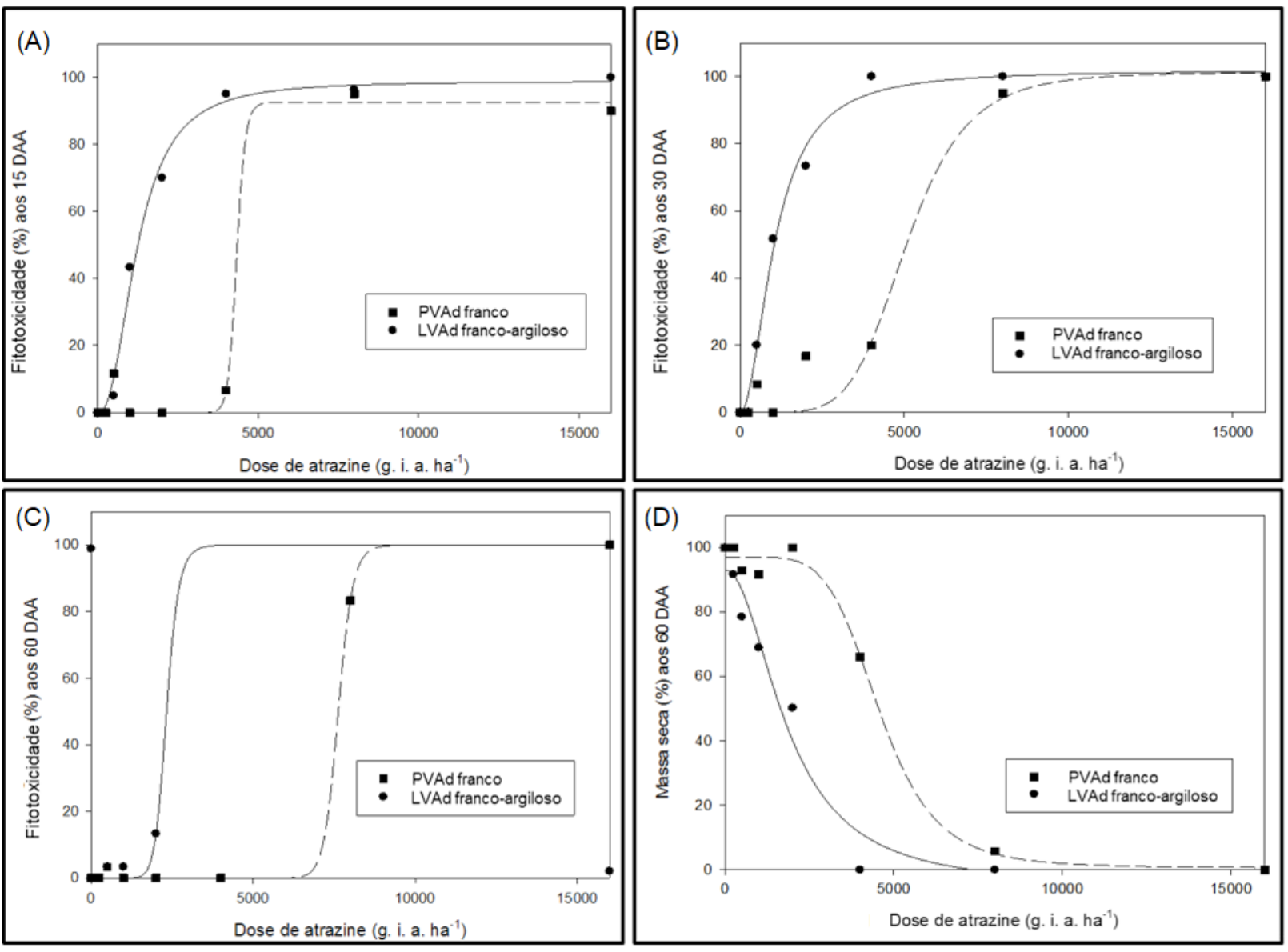

Figura 2. Fitotoxicidade (\%) de $B$. brizantha $\mathrm{cv}$. Marandu submetidas a diferentes doses de atrazine, em solo franco e franco argiloso, aos 15 (A), 30 (B) e 60 (C) dias após a aplicação (DAA) e massa seca aos 60 DAA (D) (Alta Floresta - MT, 2016).

As plantas suscetíveis à atrazine, como o capim-navalha, de acordo com Silva et al. (2013), não foram afetadas pelos herbicidas inibidores do fotossistema II, como a atrazine, até emergirem, entretanto não chegaram a lançar a primeira folha verdadeira, morrendo ainda na fase de folhas cotiledonares. No experimento com capim-navalha, na dose de $250 \mathrm{~g}^{\mathrm{h}} \mathrm{a}^{-1}$ i.a., a maioria das plantas não se desenvolveram, e a partir da dose de $500 \mathrm{~g} \mathrm{ha}^{-1}$ i.a. todas as plantas morreram ainda na fase de folhas cotiledonares pelo efeito do herbicida. Entretanto, não há registro da atrazine para pastagens no Brasil. A reforma de pastagens com a utilização da cultura do milho, cultura para qual a atrazine possui registro, pode ser uma alternativa no controle de capim-navalha, principalmente nas regiões Norte e Centro-Oeste do país, em locais com alta infestação do capim-navalha, conforme descrito por Andrade et al. (2012).

No experimento de fitotoxicidade da atrazine com o $B$. brizantha cv. Marandu, houve interação entre as fontes de variação dose e solo foi significativa $\operatorname{aos} 15(\mathrm{~F}=17,84 ; p<0,01), 30(\mathrm{~F}=9,16 ; p<0,01)$ e 60 DAA $(\mathrm{F}=23,22 ; p<0,01)$ e, para a variável massa seca aos 60 DAA $(\mathrm{F}=7,88 ; p<0,01)$ justificando o ajuste a curvas de dose-resposta.

Os parâmetros da equação log-logística (Tabela 2) para a pastagem $B$. brizantha cv. Marandu apresentaram bons ajustes dos coeficientes 
Tabela 2. Parâmetros do modelo logístico, coeficiente de determinação $\left(\mathrm{R}^{2}\right)$, teste $\mathrm{F}$, e $\mathrm{GR}_{50}$ para plantas de $B$. brizantha cv. Marandu submetidas a aplicação de diferentes doses do herbicida atrazine, em dois tipos de solos (Alta Floresta - MT, 2016).

\begin{tabular}{|c|c|c|c|c|c|c|c|}
\hline \multirow{2}{*}{ Avaliações } & \multicolumn{4}{|c|}{ Parâmetros - Modelo Logístico } & \multirow{2}{*}{$\mathbf{R}^{2}$} & \multirow{2}{*}{$\mathbf{F}^{3}$} & \multirow{2}{*}{$\mathrm{GR}_{50}{ }^{4}$} \\
\hline & Pmín' & $a$ & $\bar{b}$ & $c$ & & & \\
\hline \multicolumn{8}{|c|}{ LVAd franco argiloso } \\
\hline 15 & -- & 98,90 & 1234,62 & $-2,33$ & 0,99 & $346,22 * *$ & 1246,46 \\
\hline 30 & -- & 101,93 & 1057,15 & $-1,97$ & 1,00 & $315,21 * *$ & 1037,02 \\
\hline 60 & -- & 100,04 & 2325,30 & $-12,42$ & 1,00 & $1959,82 * *$ & 2325,16 \\
\hline $\mathrm{MS}^{2} 60$ DAA & $-5,7027$ & 98,87 & 1864,93 & 2,04 & 0,99 & $44,3740 * *$ & \\
\hline \multicolumn{8}{|c|}{ PVAd franco } \\
\hline 15 & -- & 92,50 & 4328,78 & $-32,36$ & 0,99 & $201,90 * *$ & 4350,58 \\
\hline 30 & - & 101,28 & 5089,08 & $-5,54$ & 0,99 & $92,00 * *$ & 5065,91 \\
\hline 60 & - & 100,00 & 7628,59 & $-33,85$ & 0,99 & $2838,12 * *$ & 7628,59 \\
\hline MS 60 DAA & 0,67 & 96,45 & 4593,68 & 5,54 & 0,99 & $218,34 * *$ & \\
\hline
\end{tabular}

**Valores de F significativos a 1\% de probabilidade $(p>0,01)$; ${ }^{1}$ Parâmetros do modelo logístico, de modo que Pmín é o limite inferior da curva (ponto mínimo), $a$ é a diferença entre o ponto máximo e mínimo da curva, $b$ é a dose que proporciona $50 \%$ de resposta da variável e $c$ é a declividade da curva; ${ }^{2} \mathrm{MS}=$ massa seca; ${ }^{3}$ Valores referentes a significância do modelo estatístico; ${ }^{4} \mathrm{GR}_{50}=$ dose que proporciona $50 \%$ do controle da população de plantas.

de determinação $(>90 \%)$ para todos os dias de avaliação. As doses de $\mathrm{GR}_{50}$, que proporcionaram $50 \%$ da fitotoxicidade avaliada para $B$. brizantha $\mathrm{cv}$. Marandu indicaram que para solo franco-argiloso, as doses requeridas foram menores que a dose recomendada para a atrazine $\left(2.000 \mathrm{~g} \mathrm{ha}^{-1}\right.$ i.a. $)$ aos 15 e 30 DAA, aos 60 DAA necessitou de $2325,16 \mathrm{~g} \mathrm{ha}^{-1}$ i.a. para ocasionar notas visuais de fitotoxicidade em metade da população. Em solo franco as doses de $\mathrm{GR}_{50}$ requeridas foram maiores do que $4.000 \mathrm{~g} \mathrm{ha}^{-1}$ i.a. de atrazine. Para ambos os solos houve a necessidade de doses acima de $2.000 \mathrm{~g} \mathrm{ha}^{-1}$ i.a. aos 60 DAA, sendo observada a retomada de crescimento da pastagem posteriormente aos 30 DAA.

$\mathrm{O}$ baixo teor de matéria orgânica (MO) em ambos os solos (14,2 $\mathrm{g} \mathrm{kg}^{-3}$ em PVAd franco e $18,7 \mathrm{~g} \mathrm{~kg}^{-3}$ em LVAd franco argiloso) (Tabela 1) dos experimentos indicou que a atrazine pode ter sido pouco sorvida aos solos, devido a influência da MO neste processo, e que foi possivelmente perdida por lixiviação. Quando há baixo teor de MO, a contribuição da fração argila para a sorção da atrazine pode considerada relevante (Correia e Langenbach, 2006), de modo que sua sorção seria maior no solo franco-argiloso. Para atrazine as doses são mais elevadas para solos mais argilosos e ricos em $\mathrm{MO}$, onde é pouco lixiviada em solos com teores médios e altos de argila ou MO (Archangelo et al., 2010). De modo que a sorção reduz a biodisponibilidade do produto, e consequentemente seu efeito fitotóxico para as plantas.

A curva dose-resposta (Figura 2) obtida para a pastagem B. brizantha $\mathrm{cv}$. Marandu, aos 15 e 30 DAA, indicou que a pastagem foi suscetível às doses de atrazine, evidenciando maior fitotoxicidade $(70,97 \%$ aos 15 DAA e $73,33 \%$ aos 30 DAA na dose de $2000 \mathrm{~g} \mathrm{ha}^{-1}$ de i.a.) no LVAd franco-argiloso e o PVAd franco apresentou menores danos $(0,77 \%$ aos 15 DAA e $16,66 \%$ aos 30 DAA na dose de $2000 \mathrm{~g} \mathrm{ha}^{-1}$ ) ao herbicida. Aos 60 DAA as plantas evidenciaram retomadas de crescimento, principalmente em solo de textura franca. A massa seca apresentada aos 60 DAA confirmou os resultados referentes às notas de fitotoxicidade, visto que o acúmulo de massa foi de 50,54\% para o LVAd franco-argilosa e $100 \%$ para o PVAd franco, na dose de $2000 \mathrm{~g} \mathrm{ha}^{-1}$. Ceccon et al. (2010) em seu trabalho afirmaram 
que a $B$. ruziziensis apresentou maior tolerância à atrazine (1.760 $\mathrm{g} \mathrm{ha}^{-1}$ i.a.) do que para mesotrione (60 $\mathrm{g} \mathrm{ha}^{-1}$ i.a.), não demonstrando sinais visíveis de toxicidade nas folhas para atrazine, ocasionando maior acúmulo de massa durante e após o cultivo do milho safrinha.

No experimento de fitotoxicidade da atrazine sobre a pastagem $P$. maximum cv. Mombaça a interação entre as fontes de variação dose e solo foi significativa aos $15(\mathrm{~F}=2,36 ; p<0,05)$, $30(\mathrm{~F}=5,36 ; p<0,01)$ e 60 DAA $(\mathrm{F}=1,21,12$; $p<0,01)$ e, para a variável massa seca aos 60 DAA $(\mathrm{F}=50,24 ; p<0,01)$ justificando o ajuste a curvas de dose-resposta.

Para a pastagem $P$. maximum cv. Mombaça os parâmetros da equação log-logística (Tabela 3), indicaram ajustes satisfatórios pelos coeficientes de determinação (>90\%). As doses de $\mathrm{GR}_{50}$ encontradas foram menores do que as encontradas para $B$. brizantha cv. Marandu, aos 30 e 60 DAA, sendo estas doses menores do que $2.000 \mathrm{~g} \mathrm{ha}^{-1}$ i.a. (recomendada para o herbicida).

Aos 60 DAA, houve a necessidade de maiores doses (>2.000 $\mathrm{g} \mathrm{ha}^{-1}$ i.a.) do herbicida para causar maior fitotoxicidade na população de $P$. maximum cv. Mombaça, em PVAd franco, provavelmente devido ao menor teor de argila $(28,8 \%)$ e de MO $\left(14,2 \mathrm{~g} \mathrm{~kg}^{-3}\right)$. Neste sentido, Correia e Langenbach (2006) avaliando o transporte via lixiviação de atrazine (3000 $\mathrm{g} \mathrm{ha}^{-1} \mathrm{i}$.a.) no solo, encontraram o herbicida em $50 \mathrm{~cm}$ de profundidade após $90 \mathrm{DAA}$ em solo com baixo teor de argila $(2,8 \%$ de $0-10 \mathrm{~cm}$ e $0,83 \%$ de $10-50 \mathrm{~cm}$ ).

A retomada de crescimento foi evidente aos 60 DAA, onde as doses requeridas para ocasionar danos na pastagem foram maiores, 508,83 e 2165,42 $\mathrm{g} \mathrm{ha}^{-1}$ i.a., para solo LVAd franco-argiloso e PVAd franco, respectivamente. Nas curvas dose-resposta obtidas para P. maximum cv. Mombaça (Figura 3) doses maiores que $500 \mathrm{~g} \mathrm{ha}^{-1}$ indicaram fitotoxicidade em mais de $50 \%$ da população de plantas. Aos 60 DAA as curvas se distanciaram, devido a retomada de crescimento da pastagem no solo PVAd franco, de modo que foram necessárias doses maiores do herbicida para se atingir maior fitotoxicidade. Aos 60 DAA, confirmou-se a fitotoxicidade por meio do acúmulo de massa. De modo que a pastagem de $P$. maximum cv. Mombaça foi uma alternativa a reforma de pastagens em áreas

Tabela 3. Parâmetros do modelo logístico, coeficiente de determinação $\left(\mathrm{R}^{2}\right)$, teste $\mathrm{F}$, e $\mathrm{GR}_{50}$ para plantas de $P$. maximum cv. Mombaça submetidas a aplicação de diferentes doses do herbicida atrazine, em dois tipos de solos (Alta Floresta - MT, 2016).

\begin{tabular}{|c|c|c|c|c|c|c|c|}
\hline \multirow{2}{*}{ Avaliações } & \multicolumn{4}{|c|}{ Parâmetros - Modelo Logístico } & \multirow{2}{*}{$\mathbf{R}^{2}$} & \multirow{2}{*}{$\mathbf{F}^{3}$} & \multirow{2}{*}{$\mathbf{G R}_{50}{ }^{4}$} \\
\hline & Pmín ${ }^{1}$ & $a$ & $b$ & $c$ & & & \\
\hline \multicolumn{8}{|c|}{ LVAd franco argiloso } \\
\hline 15 & -- & 98,70 & 350,14 & $-5,49$ & 1,00 & $107,48 * *$ & 351,83 \\
\hline 30 & - & 99,88 & 372,03 & $-4,70$ & 1,00 & $72.335,96 * *$ & 372,23 \\
\hline 60 & - & 100,91 & 511,24 & $-3,82$ & 1,00 & $294,9194 * *$ & 508,85 \\
\hline $\mathrm{MS}^{2} 60 \mathrm{DAA}$ & $-0,42$ & 96,82 & 533,86 & 5,98 & 0,99 & $588,51 * *$ & \\
\hline \multicolumn{8}{|c|}{ PVAd franco } \\
\hline 15 & -- & 121,96 & 519,15 & $-0,52$ & 0,99 & $107,48 * *$ & 257,88 \\
\hline 30 & -- & 102,99 & 542,14 & $-1,34$ & 0,98 & $80,40 * *$ & 519,10 \\
\hline 60 & - & 99,93 & 2164,98 & $-6,89$ & 1,00 & $154.365,28 * *$ & 2165,42 \\
\hline MS 60 DAA & $-2,59 \mathrm{E}-05$ & 96,00 & 2243,31 & 24,25 & 1,00 & $633,01 * *$ & \\
\hline $\begin{array}{l}\text { **Valores de } \mathrm{F} \\
\text { é o limite infer } \\
\text { que proporcion } \\
\text { significância do }\end{array}$ & $\begin{array}{l}\text { nificativos } \\
\text { da curva }( \\
0 \% \text { de resp } \\
\text { odelo estatí }\end{array}$ & $\begin{array}{l}\text { o de pro } \\
\text { mínin } \\
\text { da vari }\end{array}$ & $\begin{array}{l}\text { lade }(p> \\
\text { é a difer } \\
c \text { é a de }\end{array}$ & $\begin{array}{l}{ }^{1} \text { Parâm } \\
\text { ntre o } \\
\text { de da c } \\
\text { na } 50 \%\end{array}$ & áxim & $\begin{array}{l}\text { ogístico, de mo } \\
\text { nínimo da curv } \\
\text { a seca; }{ }^{3} \text { Valore } \\
\text { opulação de pla }\end{array}$ & $\begin{array}{l}\text { o que } P m \\
b \text { é a do }\end{array}$ \\
\hline
\end{tabular}



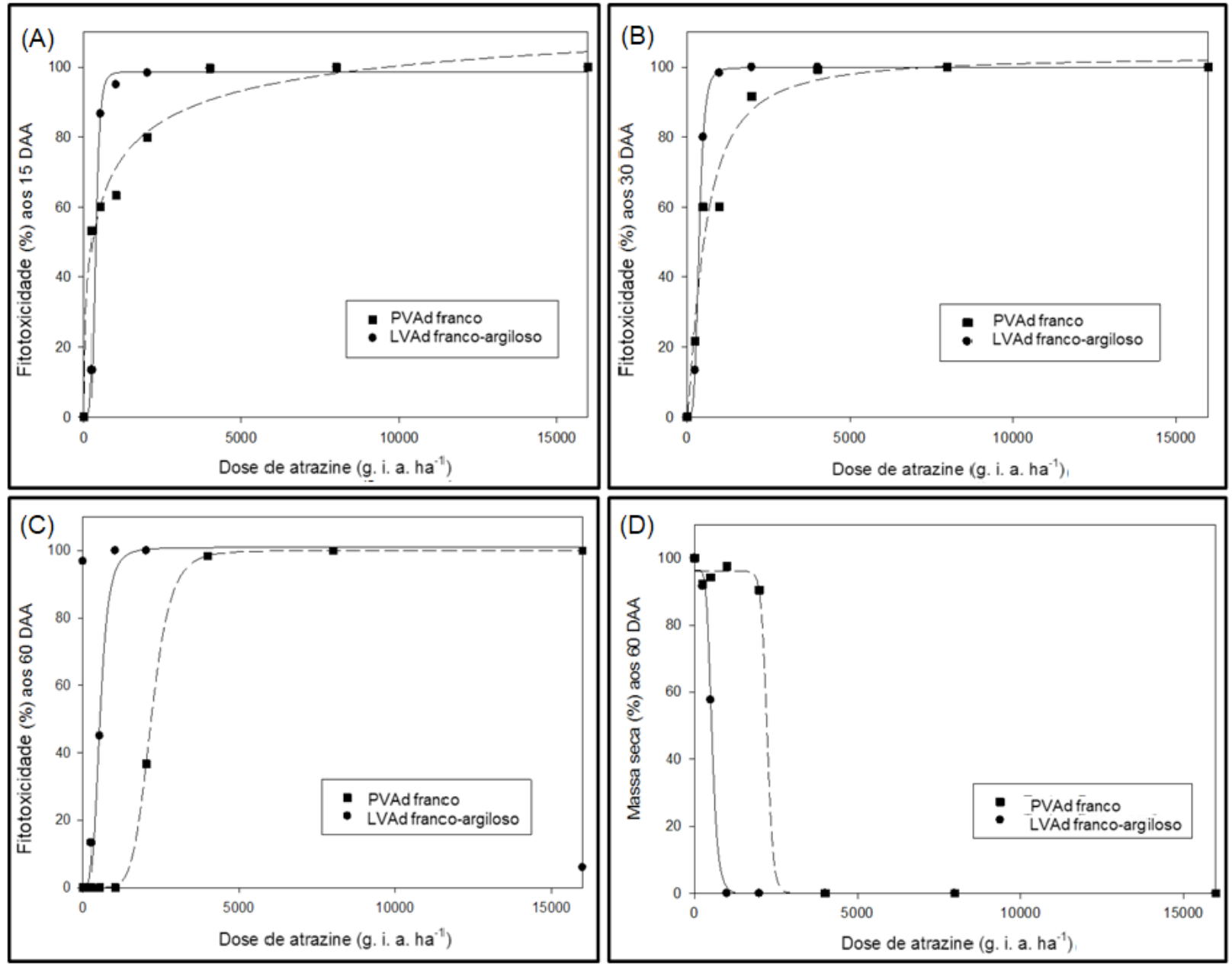

Figura 3. Fitotocixidade (\%) de P. maximum cv. Mombaça submetidas a diferentes doses de atrazine, em solo franco e franco argiloso, aos 15 (A), 30 (B) e 60 (C) dias após a aplicação (DAA) e massa seca aos 60 DAA (D) (Alta Floresta - MT, 2016).

infestadas pelo capim-navalha, em que a dose de $500 \mathrm{~g} \mathrm{ha}^{-1}$ i.a. permite recuperação da forragem e controle do capim-navalha, como demostrado anteriormente.

Outro fator que pode contribuir para a menor fitotoxicidade é a seletividade ao herbicida, que se baseia na capacidade da planta em absorver, translocar e metabolizar estes produtos, de acordo com as características de cada planta (Galon et al., 2009). Para espécies de Panicum há estudos que demonstraram a formação de metabólitos a partir da atrazine, como Thompson et al. (1971) que encontraram $44 \%$ de metabolização da atrazine para P. dichotomiflorum Michx. em 6 h após a aplicação do herbicida.

\section{Conclusões}

A atrazine, a partir da dose de $500 \mathrm{~g} \mathrm{ha}^{-1}$ i.a., demonstrou eficiência no controle de capim-navalha ( $P$. virgatum L.) em pré-emergência. Para a pastagem do gênero $B$. brizantha $\mathrm{cv}$. Marandu mais de $50 \%$ de dano nas plantas foi encontrado em doses acima da recomendada para o a atrazine (2.000 $\mathrm{g} \mathrm{ha}^{-1}$ i.a.) e para e P. maximum cv. Mombaça. Neste sentido, é possível aplicar a atrazine em 
sistemas para a reforma de pastagem em milho, pois o capim-navalha pode ser controlado efetivamente. Bem como a aplicação em pré-emergência em áreas de reforma com as pastagens $B$. brizantha cv. Marandu e P. maximum cv. Mombaça, na dose de campo para a atrazine (2.000 $\mathrm{g} \mathrm{ha}^{-1}$ i.a.), de modo que 30 DAA houve a recuperação da fitotoxicidade causada pela aplicação do produto e controle efetivo do capim-navalha presente em áreas infestadas anteriormente.

\section{Agradecimentos}

À Fundação de Amparo à Pesquisa do Estado de Mato Grosso (FAPEMAT), pelo apoio financeiro (Processo: 416251/2015).

\section{Referências}

Akbulut, G.B.; Yigit, E. The changes in some biochemical parameters in Zea mays cv. "Martha F1" treated with atrazine. Ecotoxicology and Environmental Safety, v.73, n.6, p.1429-1432, 2010. http://dx.doi.org/10.1016/j.ecoenv.2010.05.023.

ALAM - Asociación Latinoamericana de Malezas. Recomendaciones sobre unificación de los sistemas de evaluación en ensayos de control de malezas. ALAM, v.1, n.1, p.35-38, 1974.

Andrade, C.M.S.; Fontes, J.R.A.; Oliveira, T.K.; Farinatti, L.H.E. Reforma de pastagens com alta infestação de Capim-navalha (Paspalum virgatum L). Rio Branco: Embrapa Acre, 2012. 14p. (Circular Técnica, 64).

Archangelo, E.R.; Prates, H.T.; Ferreira, F.A.; Karam, D.; Ferreira, L.R.; Cardoso, A.A. Sorção, dessorção e potencial de lixiviação de atrazine em solos brasileiros. Revista Brasileira de Milho e Sorgo, v.4, n.1, p.14-27, 2010. http://dx.doi. org/10.18512/1980-6477/rbms.v4n1p14-27.

Binet, F.; Kersant'e, A.; Munier-Lamy, C.; Le Bayon, R.-C.; Belgy, M.J.; Shipitalo, M.J. Lumbricid macrofauna alter atrazine mineralization and sorption in a silt loam soil. Soil Biology \& Biochemistry, v.38, n.6, p.1255-1263, 2006. http://dx.doi.org/10.1016/j.soilbio.2005.09.018.

Carvalho, S.A.; Lima, J.M.; Curi, N.; Silva, C.A.; Toledo, J.P.V.F.; Soares, F.V. Coeficiente de distribuição do inseticida tiametoxam na fração mineral de solos sob efeito de ácidos orgânicos mono, di e tricarboxílicos. Quimica Nova, v.36, n.9, p.1323-1331, 2013. http://dx.doi.org/10.1590/ S0100-40422013000900008.

Ceccon, G.; Matoso, A.O.; Neto Neto, A.L.; Palombo, L. Uso de herbicidas no consórcio de milho safrinha com Brachiaria ruziziensis. Planta Daninha, v.28, n.2, p.359-364, 2010. http://dx.doi. org/10.1590/S0100-83582010000200015.

Correia, F.V.; Langenbach, T. Dinâmica da distribuição e degradação de atrazina em argissolo vermelho-amarelo sob condições de clima tropical úmido. Revista Brasileira de Ciência do Solo, v.30, n.1, p.183-192, 2006. http://dx.doi. org/10.1590/S0100-06832006000100018.

De la Cruz, R.; Merayo, A.; Zuñiga, H. Combate químico de Paspalum virgatum. In: Annual Meetings of PCCMCA, 36., 1990, San Salvador. Proceedings... San Salvador: PCCMCA, 1990. p.1.

Dias Filho, M.B. Degradação de pastagens: processos, causas e estratégias de recuperação 4.ed. Belém: Edição do Autor, 2011. 215p.

EWRC - European Weed Research Council. Report of 3rd and 4rd meetings of EWRC: committee of methods in weed research. Weed Research, v.4, p.88, 1964.

Galon, L.; Ferreira, E.A.; Concenço, G.; Silva, A.A.; Vargas, L. Tolerância de culturas e plantas daninhas a herbicidas. In: Agostineto, D.; Vargas, L. (Ed.). Resistência de plantas daninhas a herbicidas no Brasil. Passo Fundo: Berthier, 2009. p.37-74.

Girotto, M.; Araldi, R.; Velini, E.D.; Gomes, G.L.G.C.; Carbonari, C.A.; Jasper, S.P.; Trindade, 
M.L.B. Eficiência fotossintética da cana-de-açúcar submetida à aplicação de atrazine e tebuthiuron em pré-emergência. Revista Brasileira de Herbicidas, v.10, n.2, p.134-142, 2011. http:// dx.doi.org/10.7824/rbh.v10i2.95.

Guimarães, A.C.D.; Takeshita, V.; Catissi, F.; Nicolai, M.; Christoffoleti, P.J. Eficácia do imazapic no controle de capim-camalote aplicado em solos de diferentes texturas. Revista Brasileira de Herbicidas, v.15, n.3, p.213-220, 2016. http:// dx.doi.org/10.7824/rbh.v15i3.450.

Kluthcouski, J.; Yokoyama, L.P. Opções de integração lavoura-pecuária. In: Kluthcouski, J.; Stone, L.F.; Aidar, H. (Ed.). Integração lavourapecuária. Santo Antônio de Goiás: Embrapa Arroz e Feijão, 2003. cap.4, p.131-141.

Loch, D.S.L.; Harvey, G.L. Developing herbicide strategies for tropical herbage seed crops. In: Australian New Crops Conference, 1., 1996, Queensland. Proceedings... Queensland: Gaton College, 1997. p.273-282.

Merini, L.J.; Bobillo, C.; Cuadrado, V.; Corach, D.; Giulietti, A.M. Phytoremediation potential of the novel atrazine tolerant Lolium multiflorum and studies on the mechanisms involved. Environmental Pollution, v.157, n.11, p.3059-3063, 2009. http:// dx.doi.org/10.1016/j.envpol.2009.05.036.

Produção da Pecuária Municipal. Rio de Janeiro: IBGE, 2016. v.44. Disponível em: <https:// biblioteca.ibge.gov.br/index.php/bibliotecacatalogo $? \mathrm{id}=784 \&$ view $=$ detalhes $>$. Acesso em: 25 jan. 2018.

Scorza Júnior, R.P.; Rigitano, R.L.O. Sorção, degradação e lixiviação inseticida tiametoxam em dois solos de Mato Grosso do Sul. Revista Brasileira de Engenharia Agrícola e Ambiental, v.16, n.5, p.564-572, 2012. http://dx.doi.org/10.1590/ S1415-43662012000500013.

Seefeldt, S.S.; Jensen, S.E.; Fuerst, E.P. Log-logistic analysis of herbicide dose response relationship.
Weed Technology, v.9, n.2, p.218-227, 1995. http://dx.doi.org/10.1017/S0890037X00023253.

Silva, A.A.; Vivian, R.; Oliveira Júnior, R.S. Herbicidas: comportamento no solo. In: Silva, A.; Silva, J.F. (Ed.). Tópicos em manejo de plantas daninhas. Viçosa: Universidade Federal de Viçosa, 2007. p.189-248.

Silva, I.P.D.F.; Silva Junior, J.F.D.; Putti, F.F.; Latorre, D.D.O.; Schimidt, A.P.; Ludwig, R. Herbicidas inibidores do fotossistema II-parte I/ photosystem II inhibitor herbicides-part I. Revista Brasileira de Engenharia de Biossistemas, v.7, n.1, p.1-11, 2013. http://dx.doi.org/10.18011/ bioeng2013v7n1p1-11.

Silva, L.S.; Franco, M.P.E.; Souza, F.R.S. Controle químico do capim-navalha. In: Simpósio Nacional sobre Plantas Daninhas em Sistemas de Produção Tropical, 2.; Simpósio Internacional Amazônico sobre Plantas Daninhas, 5., 2017, Alta Floresta. Anais... Alta Floresta: SBCPD, 2017. 93p.

Silva, W.J.; Yamashita, O.M.; Silva, P.C.L.; Felito, R.A.; Rocha, A.M.; Ferreira, A. Quebra de dormência de sementes de capim-navalhão. Revista da Universidade Vale do Rio Verde, v.15, n.2, p.830-842, 2018.

Sistachs, C.M.; Fernandez, L.; León, J.J. Susceptibilidad de la semilla de caguazo (Paspalum virgatum L.) a diferentes herbicidas. Revista Cubana de Ciências Agrícolas, v.16, p.113-117, 1982.

Sistachs, C.M.; León, J.J. El caguazo (Paspalum virgatum L.): aspectos biológicos, su control en pastizales. Havana: Edica, 1987. 57p.

Streibig, J.C.; Rudemo, M.; Jensen, J.E. Doseresponse curves and statistical models. In: Streibig, J.C.; Kudsk, P. (Ed.). Herbicide bioassay. Boca Raton: CRC Press, 1993. p.30-35.

Thompson, L.; Houghton, J.; Slife, F.; Butler, H. Metabolism of atrazine by fall panicum and large crabgrass. Weed Science, v.19, n.4, p.409-412, 1971. 
Valls, J.F.M.; Oliveira, R.C. Paspalum. In: Jardim Vilela, L.; Souza, D.M.G.; Silva, J.E. Calagem Botânico do Rio de Janeiro - JBRJ. Lista de e adubação para pastagens. In: Souza, D.M.G; espécies da flora do Brasil. Rio de Janeiro: JBRJ, Lobato, E. (Ed.). Cerrado: correção do solo e 2012. Disponível em: <http://floradobrasil.jbrj. adubação. 2.ed. Brasília: Embrapa Informação gov.br/2012/FB020485>. Acesso em: 27 jul. 2012. Tecnológica, 2004. p.169-183. 